\section{Padrão alimentar, asma e sibilo atópico e não atópico em crianças e adolescentes: estudo SCAALA, Salvador, Bahia, Brasil}

\author{
Dietary pattern, asthma, and atopic and non-atopic \\ wheezing in children and adolescents: SCAALA \\ study, Salvador, Bahia State, Brazil \\ Patrón dietético, asma y sibilancias atópicas y no \\ atópicas en niños y adolescentes: estudio \\ SCAALA, Salvador, Bahía, Brasil
}

\begin{abstract}
A cross-sectional study was conducted on dietary patterns and their influence on the occurrence of wheezing and atopic and non-atopic asthma in a sample of 1,168 children and adolescents in Salvador, Bahia State, Brazil. Wheezing and asthma symptoms in the previous 12 months were obtained using the ISAAC questionnaire. The presence of aeroallergen-specific IgE was identified. A food frequency questionnaire was used to define dietary patterns. The study applied logistic regression and multinomial polytomous logistic regression. Fish consumption was associated with a $27 \%$ reduction in wheezing (95\%CI: 0.56-0.94), 37\% in asthma (95\%CI: 0.47-0.83), 51\% in non-atopic asthma (95\%CI: 0.31-0.79), and 38\% in non-atopic wheezing (95\%CI: 0.46-0.83). The highest tertile of dietary patterns reduced wheezing by 27\% (95\%CI: 0.570.95), atopic wheezing by 46\% (95\%CI: 0.30 0.98), asthma by 36\% (95\%CI: 0.49-0.83), and atopic asthma by 50\% (95\%CI: 0.28-0.89). Fish consumption may thus have a protective effect against wheezing and non-atopic asthma and dietary pattern against atopic asthma and wheezing.
\end{abstract}

Feeding Behavior; Asthma; Respiratory Sounds

\author{
Silvana D'Innocenzo 1 \\ Sheila M. A. Matos 1 \\ Matildes S. Prado 1 \\ Carlos A. S. T. Santos 2 \\ Ana M. O. Assis 3 \\ Alvaro A. Cruz 4 \\ Dirce M. L. Marchioni 5 \\ Laura C. Rodrigues 6 \\ Maurício L. Barreto 1
}

\section{Resumo}

Avaliou-se, em estudo transversal, a influência do padrão alimentar sobre a ocorrência de sibilo e asma atópica e não atópica em 1.168 crianças e adolescentes em Salvador, Bahia, Brasil. Sibilo e sintomas de asma nos últimos 12 meses foram obtidos por meio do questionário ISAAC. Atopia foi definida pela presença de IgE específica a aerolérgenos. Questionário de frequência alimentar foi usado para definir padrões alimentares. Empregou-se regressões logística e logística politômica multivariadas. O consumo de pescados reduziu a ocorrência de sibilo em 27\% (IC95\%: 0,56-0,94) e asma em 37\% (IC95\%: 0,47-0,83); asma não atópica em 51\% (IC95\%: 0,31-0,79) e sibilo não atópico em 38\% (IC95\%: 0,46-0,83). O maior tercil do padrão alimentar reduziu o sibilo em 27\% (IC95\%: 0,57-0,95), sibilo atópico em 46\% (IC95\%: 0,30-0,98), asma em 36\% (IC95\%: 0,49-0,83) e asma atópica em 50\% (IC95\%: 0,280,89). O consumo de pescados pode conferir efeito protetor para sibilo e asma não atópica e o padrão alimentar para sibilo e asma atópica.

Comportamento Alimentar; Asma; Sons

Respiratórios 


\section{Introdução}

Asma tem sido um importante problema de saúde pública entre crianças nas mais diversas zonas geográficas do mundo, com aumento da ocorrência tanto em países desenvolvidos quanto em desenvolvimento 1. Na América Latina a maior prevalência de asma entre as crianças é não atópica e tem sido associada aos fatores relacionados à pobreza, e entre eles destacam-se precárias condições de higiene, estresses psicossociais, obesidade e qualidade da dieta 2 . Em Salvador (Brasil), a prevalência de sibilância nos últimos 12 meses foi de $24,6 \%$ em crianças de 13 a 14 anos e de $17,2 \%$ para crianças de 6 a 7 anos, sendo que os percentuais para crianças do Nordeste brasileiro foram de $20,3 \%$ para as maiores (13 a 14 anos) e de $21,0 \%$ para as menores (6 a 7 anos) 3 . Dados do SCAALA (Social Changes, Asthma and Allergy in Latin America) mostram que, em Salvador, a maior parte da ocorrência da asma é persistente 4 , a prevalência de sibilância entre crianças atópicas foi de $34,8 \%$ e de $25 \%$ entre as não atópicas 5 , sendo que crianças atópicas apresentaram $60 \%$ mais chances de desenvolver sibilância, comparadas às não atópicas. O número de internações e de atendimentos em emergência é relevante e pode sofrer redução significativa com atenção e cuidado específico em programas de controle de asma ${ }^{6}$. Esse panorama sinaliza a asma como um importante problema de saúde pública para Salvador.

Nas últimas décadas, o estilo de vida ocidental tem sido associado com complexas mudanças ambientais, comportamentais, e dietéticas, as quais têm sido apontadas como aspectos importantes na etiologia da asma 3,7,8,9. A transição à dieta ocidental pode refletir o consumo reduzido em componentes antioxidantes da dieta como vitamina $\mathrm{A}$, carotenóides, vitamina $\mathrm{C}$, vitamina E, zinco, selênio, cobre e compostos bioativos, os quais têm potencial ação protetora no sistema respiratório e na redução da ocorrência de asma $9,10,11$. O padrão dietético ocidental dominante caracteriza-se ainda pelo maior consumo de gorduras saturadas e menor consumo de ácido graxo poli-insaturado ômega 3 ( $n$-3-PUFA), aumentando o risco de ocorrência de asma 9,12,13. Existem evidências de que a adesão ao padrão Mediterrâneo (composto por peixes, frutas, vegetais, cereais integrais, com alto conteúdo de antioxidantes e $n$-3-PUFA), tem efeito protetor para a ocorrência da asma 11,13,14.

Acumulam-se evidências científicas sobre os possíveis mecanismos imbricados na relação dieta e asma. Argumentos teóricos na direção da hipótese lipídica e antioxidante são desenvolvidos por Devereux \& Seaton 9. O papel da dieta também tem sido relacionado a mecanismos complexos na modulação da resposta imune adquirida ou inata 15 , variando de acordo aos componentes lipídicos e os antioxidantes da dieta.

Tradicionalmente os estudos que avaliam a dieta e sua relação com as enfermidades consideravam os efeitos de alimentos ou nutrientes de modo isolados, entretanto, o ato de se alimentar abarca uma complexidade cuja representação do consumo pode ser bem evidenciada em estudos que adotam os padrões dietéticos 15,16, os quais refletem o consumo habitual de alimentos e nutrientes, além de constituírem em abordagem para a avaliação da relação entre dieta e doenças crônicas $16,17,18$. Assim, ratifica-se a importância de avaliar aspectos que possam interferir na prevalência da doença, tais como o padrão alimentar.

A relação dieta e asma pode ser acessada sob a ótica de diferentes abordagens na epidemiologia nutricional 15,16, e este estudo contemplou duas delas: padrão alimentar e grupos de alimentos.

O objetivo deste trabalho foi avaliar a influência do padrão alimentar e cada grupo de alimento que o compõe sobre a ocorrência de sibilo atópico e não atópico, e asma atópica e não atópica em crianças/adolescentes de um grande centro urbano brasileiro.

\section{Metodologia}

Trata-se de estudo transversal de base populacional com crianças e adolescentes de 4 a 11 anos de idade da cidade de Salvador, participantes do projeto SCAALA, realizado nos anos de 2005 e 2006. Detalhes sobre o desenho e seleção da amostra podem ser obtidos em publicação anterior 19. O projeto SCAALA foi composto por 1.445 crianças que foram aleatoriamente selecionadas de 20 mil domicílios de níveis socioeconômicos e condições ambientais variadas 19. A amostra deste estudo compreendeu 1.168 crianças que dispunham de informações completas para as variáveis estudadas.

Informações referentes aos sinais e sintomas compatíveis com asma foram obtidas com os pais ou responsáveis, empregando-se questionário padronizado adaptado da versão em português do questionário usado pelo International Study of Asthma and Allergies in Childhood (ISAAC), segunda fase 20. Definiu-se sibilo com base no relato positivo à presença de sibilo nos últimos 12 meses. Asma foi definida com base na presença de sibilo nos últimos 12 meses e adição de pelo menos um dos seguintes aspectos: ter apresentado asma alguma vez na vida; ter observado sibilo ou piado no peito ao respirar 
durante ou depois de fazer exercício, nos últimos 12 meses; ter apresentado pelo menos quatro crises (ataques) de sibilo no peito, nos últimos 12 meses; ter acordado pelo menos uma noite por semana por causa do sibilo, nos últimos 12 meses. Esses critérios usados são mais específicos que sibilo nos últimos 12 meses, conforme utilizado pelo ISAAC conferindo maior robustez à definição de asma 5 .

Amostras de sangue foram coletadas para medir IgE específica a alérgenos empregandose radioimunoensaio (RAST) utilizando-se os kits comerciais e o teste ELISA. Maiores detalhes podem ser encontrados em Barreto et al. 19. Atopia foi definida pela presença de IgE específica para pelo menos um dos seguintes aeroalérgenos (Periplaneta americana, Dermatophagoides pteronyssinus, Blomia tropicalis e Blatela germâni$c a$, cão e gato). Considerou-se o ponto de corte $0,70 \mathrm{KU} / \mathrm{l}$. Valores abaixo desses limites eram indicativos de não detecção.

A combinação das variáveis sibilo, asma e atopia permitiu a classificação das crianças em: participantes com sibilo atópico, os quais apresentaram relato positivo para sibilo e concentrações de IgE detectáveis; já os definidos com sibilo não atópico apresentaram relato positivo para sibilo e concentrações de IgE não detectáveis; os asmáticos atópicos foram os que apresentaram asma e níveis de IgE detectáveis; os asmáticos não atópicos apresentaram asma e níveis de IgE não detectáveis.

As variáveis dependentes (sibilo e asma) foram conformadas por quatro categorias: asma atópica, asma não atópica, somente atópicos e saudáveis (ausência de asma, sibilo ou atopia); sibilo atópico, sibilo não atópico, somente atópicos e saudáveis (ausência de asma, sibilo ou atopia).

Informações sobre consumo alimentar foram obtidas das mães ou responsáveis pelas crianças por nutricionistas e acadêmicos de nutrição treinados.

As variáveis de consumo alimentar adotadas para este trabalho são provenientes da análise fatorial realizada em estudo anterior 21 , utilizandose o método de Análise de Componentes Principais, o qual gerou o padrão alimentar e grupos de alimentos que o compõe, com base em questionário de frequência alimentar (QFA) semiquantitativo, validado por Matos et al. 22, composto por 98 alimentos, referente ao consumo alimentar nos últimos 12 meses. Detalhes sobre a análise para definição do padrão alimentar e dos grupos de alimentos característicos dessa amostra estudada podem ser encontrados no trabalho de D'Innocenzo et al. ${ }^{21}$. No entanto, dispõe-se a seguir de breve metodologia para a definição dessas variáveis de consumo alimentar.
O referido questionário era composto por escala de frequência alimentar abarcando cinco categorias, assim representadas: nunca consome, consome de uma a três vezes por mês, consome de uma a duas vezes por semana, consome de três a quatro vezes por semana, consome cinco ou mais vezes por semana. Os dados da frequência alimentar foram transformados em fração diária de consumo com o objetivo de se utilizar apenas uma unidade temporal. Para tanto, foi calculado o ponto médio de cada categoria de frequência de consumo contida no questionário para cada alimento, dividindo-se por sete para o consumo semanal ou trinta para o consumo mensal. Posteriormente, os alimentos passaram a constituir grupos de alimentos com base nas características nutricionais e correlações obtidas por meio de análise fatorial preliminar.

Cada grupo de alimento foi sintetizado em um único valor para cada criança, conformando uma medida resumo, expressa pela soma das frações diárias de cada alimento que constitui o grupo. Foi possível, dessa forma, obter uma variável contínua que foi padronizada, segundo escore $z$ da curva normal padrão, e constitui a variável de entrada para proceder à análise fatorial por componentes principais, para a extração dos padrões alimentares.

Para este estudo foram adotados os grupos de alimentos que compuseram o padrão alimentar de maior explicação da variabilidade da dieta: preparação à base de pescados (que inclui além dos mariscos, crustáceos e peixes, outros alimentos utilizados em sua preparação, em especial, leite de coco e azeite de dendê), vegetais/tubérculos (tomate, cenoura, abóbora, batata-inglesa e batata-doce), frutas (abacaxi, laranja, limão, lima, acerola, caju, umbu, maçã, morango, cajá, caqui, melancia, goiaba, manga, ameixa, mamão comum, mamão papaia, melão, abacate, banana, uva, sucos de fruta, coco, kiwi, ameixa fresca, nozes, castanha, cana-de-açúcar, caldo de cana), leguminosas (amendoim cozido, amendoim torrado, soja texturizada e leite de soja), cereais (arroz, macarrão, bolo, biscoitos, pão francês, pão-de-leite, pão-de-milho, aveia, milho, farinha de milho, milho branco, milho em conserva, sucrilhos, farinha de cremogema, arroz integral, pão integral).

Assim, as variáveis independentes principais para este trabalho são o padrão alimentar de maior explicação da variabilidade da dieta e os grupos de alimentos que o compõe. A soma das frações diárias para cada grupo de alimento constituiu a variável independente principal para o presente estudo, categorizada em tercis (1o tercil, menor consumo; 2o tercil, consumo moderado; 3o tercil, maior consumo). O escore 
do padrão alimentar adotado também constituiu variável independente principal, expressa em tercis.

Estimou-se ainda a disponibilidade de energia (Kcal) de cada QFA para ajuste energético das análises 16. Foram excluídos os valores extremos, abaixo de $500 \mathrm{Kcal}$ e acima de $6.000 \mathrm{Kcal} 23$.

Todas as medições foram realizadas em dupla série, por profissionais e estudantes de nutrição devidamente treinados, admitindo-se variação de $100 \mathrm{~g}$ e de $0,1 \mathrm{~cm}$, respectivamente, para peso e comprimento/altura, e a média entre as duas medições foi considerada a medida final. Foram observados as normas e os critérios técnicos recomendados 24,25 em todas as etapas da avaliação antropométrica. As crianças foram pesadas em balanças microeletrônicas, portáteis (Filizola, modelo E-150/3P, São Paulo, Brasil), com capacidade de $150 \mathrm{~kg}$ e precisão de $100 \mathrm{~g}$, fornecidas pelo extinto Instituto Nacional de Alimentação e Nutrição do Ministério da Saúde (INAN/MS), e medidas usando-se infantômetro de madeira (Leiscester Height Measure). Os instrumentos foram calibrados periodicamente. As idades das crianças foram registradas usando-se a certidão de nascimento ou Cartão da Criança, e foram calculadas as diferenças entre a data de medição e a data de nascimento.

Calculou-se o índice de massa corporal (IMC) sendo consideradas com excesso de peso as crianças com IMC maior ou igual a +1 escores- $Z$, tendo como padrão de referência aquele da Organização Mundial da Saúde (OMS) em 200624 e em 200725 .

As fezes foram examinadas adotando-se as técnicas parasitológicas de sedimentação espontânea 26 e Kato-Katz 27. Considerou-se como resultado positivo, para infecção pelos helmintos intestinais Ascaris lumbricoides e Trichuris trichiura, a presença de ovos destes helmintos em qualquer um ou em ambos os exames.

As variáveis socioeconômicas (escolaridade materna, posse de bens e utensílios domésticos) deram origem a um indicador socioeconômico, inicialmente idealizado por Alvarez et al. 28 e, posteriormente, adaptado por Issler \& Giugliani 29 para a realidade brasileira. Assim, cada variável recebeu uma pontuação que variou de 0 a 4 , sendo o valor zero indicativo de pior situação socioeconômica 21. Após a somatória de todos os valores, o indicador obtido foi classificado em níveis socioeconômicos baixo e alto.

Para o presente estudo foram selecionadas segundo a literatura as seguintes variáveis de ajuste: sexo, faixa etária e índice de massa corpórea da criança, indicador socioeconômico, história de asma dos pais, história de fumo no domicílio, água canalizada e destino dos dejetos no domicílio, faixa etária da mãe, energia (Kcal) obtida por meio do QFA, infecção por A. lumbricoides e infecção por T. trichiura.

\section{Análise estatística}

Estimou-se a prevalência dos desfechos estudados (asma: atópica e não atópica; sibilo: atópico e não atópico) e aplicou-se o teste $\chi^{2}$ de tendência.

Regressão logística multivariada foi usada para testar associações entre tercis das variáveis de exposição e sibilo e asma em geral.

A regressão multinomial ${ }^{30}$ permitiu a obtenção da medida de odds ratio (OR) bruta e ajustada, com respectivo intervalo de $95 \%$ de confiança (IC95\%), da associação entre os tercis dos grupos de alimentos e desfechos de sibilo atópico e não atópico e asma atópica e não atópica, aplicandose um modelo para cada grupo alimentar considerado. Modelo similar foi elaborado para a análise de associação com o padrão alimentar e os desfechos estudados. Os modelos foram ajustados por variáveis potencialmente confundidoras.

Crianças saudáveis constituíram o grupo de referência para análises (modelo multinomial) tanto para asmáticos não atópicos quanto para sibilo não atópico. Crianças com apenas atopia (concentrações de IgE detectável sem a ocorrência de sibilo ou asma) constituíram o grupo de referência para análises (modelo multinomial) tanto para crianças asmáticas atópicas quanto para crianças com sibilo atópico.

O pacote estatístico adotado para entrada dos dados foi o Epi Info, versão 6.4 (Centers for Disease Control and Prevention, Atlanta, Estados Unidos) e as análises foram realizadas utilizando-se o software Stata, versão 9.0 (Stata Corp., College Station, Estados Unidos).

Este estudo foi aprovado pelo Comitê $\mathrm{Na}$ cional de Ética em Pesquisa, e a participação de cada criança se deu após assinatura de consentimento livre e esclarecido pelo responsável legal.

\section{Resultados}

Na Tabela 1, tem-se a caracterização da amostra, segundo variáveis de interesse. Predominaram meninos, crianças com baixo indicador socioeconômico, menores de 8 anos, filhos de pai e mãe que não tiveram asma, crianças que dispõem de rede de esgoto e água canalizada no domicílio, não infectadas por $A$. lumbricoides ou por T. trichiura, crianças eutróficas e com ausência de fumo no domicílio. Nota-se sibilo em $29,4 \%$ das crianças, asma em 23,3\% e atopia em 38,1\%.

Na Tabela 2, do total de 1.160 crianças com informações disponíveis para sibilo e atopia, fo- 
Tabela 1

Caracterização da amostra estudada de 1.168 crianças, segundo variáveis de interesse. Salvador, Bahia, Brasil, 2005-2006.

\begin{tabular}{|c|c|c|}
\hline Variáveis & $\mathrm{n}$ & $\%$ \\
\hline \multicolumn{3}{|l|}{ Sexo } \\
\hline Masculino & 626 & 53,6 \\
\hline Feminino & 542 & 46,4 \\
\hline \multicolumn{3}{|l|}{ Idade (anos) * } \\
\hline$\geq 5$ e $<6$ & 232 & 19,9 \\
\hline$\geq 6$ e $<7$ & 239 & 20,4 \\
\hline$\geq 7$ e $<8$ & 227 & 19,4 \\
\hline$\geq 8$ e $<9$ & 233 & 20,0 \\
\hline$\geq 9 \mathrm{e}<12$ & 237 & 20,3 \\
\hline \multicolumn{3}{|l|}{ Indicador socioeconômico } \\
\hline Baixo & 601 & 51,5 \\
\hline Alto & 567 & 48,5 \\
\hline \multicolumn{3}{|l|}{ Asma dos pais } \\
\hline Pai e mãe não tiveram asma & 1.014 & 86,8 \\
\hline Pai ou mãe tiveram asma & 154 & 13,2 \\
\hline \multicolumn{3}{|l|}{ Destino dos dejetos } \\
\hline Sem rede de esgoto & 192 & 16,4 \\
\hline Com rede de esgoto & 976 & 83,6 \\
\hline \multicolumn{3}{|l|}{ Água canalizada no domicílio } \\
\hline Sim & 1.076 & 92,1 \\
\hline Não & 92 & 7,9 \\
\hline \multicolumn{3}{|l|}{ Infecção por Ascaris lumbricoides } \\
\hline Negativo & 978 & 83,7 \\
\hline Positivo & 190 & 16,3 \\
\hline \multicolumn{3}{|l|}{ Infecção por Trichuris trichiura } \\
\hline Negativo & 1.000 & 85,6 \\
\hline Positivo & 168 & 14,4 \\
\hline \multicolumn{3}{|l|}{ Fumo no domicílio } \\
\hline $\operatorname{Sim}$ & 316 & 27,0 \\
\hline Não & 852 & 73,0 \\
\hline \multicolumn{3}{|l|}{ IMC da criança } \\
\hline Sobrepeso/Obesidade & 174 & 14,9 \\
\hline Eutrofia & 958 & 82,0 \\
\hline Magreza & 36 & 3,1 \\
\hline \multicolumn{3}{|l|}{ Idade da mãe (anos) } \\
\hline$<29$ & 389 & 33,3 \\
\hline$\geq 29$ e $<36$ & 396 & 33,9 \\
\hline$\geq 36$ & 383 & 32,8 \\
\hline \multicolumn{3}{|l|}{ Sibilo } \\
\hline Sim & 343 & 29,4 \\
\hline Não & 825 & 70,6 \\
\hline \multicolumn{3}{|l|}{ Asma } \\
\hline Sim & 272 & 23,3 \\
\hline Não & 896 & 76,7 \\
\hline \multicolumn{3}{|l|}{$\lg E(K U / L) * *$} \\
\hline$<0,70$ & 718 & 61,9 \\
\hline$\geq 0,70$ & 442 & 38,1 \\
\hline
\end{tabular}

IMC: índice de massa corporal.

* Quintil;

** $N=1.160$ crianças. 
Tabela 2

Prevalência dos desfechos estudados, segundo variáveis de consumo alimentar. Salvador, Bahia, Brasil, 2005-2006.

\begin{tabular}{|c|c|c|c|c|c|c|c|c|c|c|}
\hline \multirow{4}{*}{$\begin{array}{l}\text { Grupos de alimentos/ } \\
\text { Intervalo do tercil }\end{array}$} & \multicolumn{5}{|c|}{ Asma ( $N=1,130)$} & \multicolumn{5}{|c|}{ Sibilo $(N=1,160)$} \\
\hline & \multirow[t]{3}{*}{$\mathbf{n}$} & \multirow{2}{*}{\multicolumn{2}{|c|}{$\begin{array}{l}\text { Atópica * } \\
(n=129)\end{array}$}} & \multirow{2}{*}{\multicolumn{2}{|c|}{$\begin{array}{l}\text { Não atópica ** } \\
(n=140)\end{array}$}} & \multirow[t]{3}{*}{$\mathbf{n}$} & \multirow{2}{*}{\multicolumn{2}{|c|}{$\begin{array}{l}\text { Atópico *** } \\
(n=159)\end{array}$}} & \multirow{2}{*}{\multicolumn{2}{|c|}{$\begin{array}{l}\text { Não atópico \# } \\
(n=181)\end{array}$}} \\
\hline & & & & & & & & & & \\
\hline & & Prevalência & Valor de $p$ & Prevalência & Valor de $p$ & & Prevalência & Valor de $p$ & Prevalência & Valor de $p$ \\
\hline \multicolumn{11}{|l|}{ Pescados \#\# } \\
\hline T1 $(-0,10<-0,30)$ & 382 & 13,4 & & 15,5 & & 393 & 15,8 & & 18,3 & \\
\hline $\mathrm{T} 2(-0,30<0,18)$ & 375 & 11,2 & & 11,5 & & 386 & 13,7 & & 13,2 & \\
\hline T3 $(0,18-6,94)$ & 373 & 9,7 & 0,110 & 10,2 & 0,028 & 381 & 11,6 & 0,088 & 15,2 & 0,230 \\
\hline \multicolumn{11}{|l|}{ Vegetais/Tubérculos } \\
\hline T1 $(-1,37<-0,46)$ & 402 & 12,7 & & 12,2 & & 414 & 15,2 & & 15,0 & \\
\hline T2 $(-0,46<0,35)$ & 378 & 12,2 & & 11,1 & & 387 & 14,2 & & 14,2 & \\
\hline T3 $(0,35-5,1)$ & 350 & 9,1 & 0,134 & 14,0 & 0,475 & 359 & 11,4 & 0,130 & 17,8 & 0,294 \\
\hline \multicolumn{11}{|l|}{ Frutas } \\
\hline $\mathrm{T} 1(-1,77<-0,52)$ & 382 & 13,1 & & 11,3 & & 392 & 15,3 & & 15,3 & \\
\hline T2 $(-0,52<0,33)$ & 372 & 12,1 & & 13,4 & & 384 & 14,8 & & 16,4 & \\
\hline T3 $(0,33-4,0)$ & 376 & 9,0 & 0,080 & 12,5 & 0,601 & 384 & 10,9 & 0,078 & 15,1 & 0,941 \\
\hline \multicolumn{11}{|l|}{ Leguminosas } \\
\hline $\mathrm{T} 1(-0,64<-0,32)$ & 494 & 12,4 & & 13,0 & & 509 & 14,9 & & 16,1 & \\
\hline $\mathrm{T} 2(-0,32<0,33)$ & 322 & 11,8 & & 8,7 & & 332 & 14,5 & & 11,8 & \\
\hline T3 $(0,33-6,99)$ & 313 & 9,6 & 0,245 & 15,0 & 0,564 & 318 & 11,0 & 0,128 & 18,6 & 0,514 \\
\hline \multicolumn{11}{|l|}{ Cereais } \\
\hline $\mathrm{T} 1(-2,57<-0,46)$ & 387 & 12,9 & & 12,7 & & 393 & 14,3 & & 16,3 & \\
\hline $\mathrm{T} 2(-0,46<0,37)$ & 373 & 12,1 & & 13,1 & & 387 & 15,3 & & 16,5 & \\
\hline T3 $(0,37-4,66)$ & 370 & 9,2 & 0,108 & 11,4 & 0,589 & 380 & 11,6 & 0,285 & 14,0 & 0,374 \\
\hline \multicolumn{11}{|l|}{ Padrão alimentar } \\
\hline $\mathrm{T} 1(-2,27<-0,48)$ & 371 & 14,0 & & 12,9 & & 380 & 16,1 & & 16,6 & \\
\hline $\mathrm{T} 2(-0,48<0,30)$ & 381 & 11,8 & & 12,3 & & 394 & 14,7 & & 14,0 & \\
\hline T3 $(0,30-5,19)$ & 377 & 8,5 & 0,018 & 11,7 & 0,598 & 385 & 10,4 & 0,023 & 16,1 & 0,859 \\
\hline \multicolumn{11}{|c|}{ Sibilo nos últimos 12 meses + sintomas + IgE $\geq 0,70 \mathrm{KU} / \mathrm{L}$} \\
\hline \multicolumn{11}{|c|}{ ** Sibilo nos últimos 12 meses + sintomas + IgE < 0,70KU/L; } \\
\hline \multicolumn{11}{|c|}{ *** Sibilo nos últimos 12 meses $+\lg E \geq 0,70 \mathrm{KU} / \mathrm{L}$} \\
\hline \multicolumn{11}{|c|}{ \# Sibilo nos últimos 12 meses + lgE <0,70KU/L; } \\
\hline * Preparação à base $\mathrm{d}$ & & & & & & & & & & \\
\hline
\end{tabular}

ram estudadas 159 crianças $(13,7 \%)$ com sibilo atópico 181 (15,6\%) com sibilo não atópico, 537 (46,3\%) saudáveis e 283 (24,4\%) com apenas atopia. Dentre as 1.130 crianças com informações disponíveis para asma e atopia, foram estudadas 129 (11,4\%) com asma atópica, $140(12,4 \%)$ com asma não atópica, 578 (51,2\%) saudáveis e 283 (25\%) com apenas atopia. Observa-se a prevalência dos desfechos estudados, segundo variáveis de consumo alimentar. Houve diminuição na prevalência de asma não atópica com o aumento do consumo do grupo com preparação à base de pescados $(p=0,028)$. Nos mais elevados tercis de consumo do padrão alimentar ocorreu redução tanto na prevalência de asma atópica ( $p=0,018)$ quanto no sibilo atópico $(\mathrm{p}=0,023)$.
A Tabela 3 mostra o resultado da associação entre variáveis de consumo alimentar e sibilo em geral, sibilo atópico e não atópico. Verificou-se que o maior tercil de consumo de alimentos do grupo com preparação à base de pescados apresentou efeito protetor, reduzindo a ocorrência de sibilo, em geral, em 27\% (OR = 0,73; IC95\%: 0,56$0,94)$ e no segundo tercil de consumo, para sibilo não atópico, em 38\% (OR = 0,62; IC95\%: 0,460,83 ), resultados mantidos mesmo após ajuste. O maior tercil de consumo do padrão alimentar também reduziu a ocorrência de sibilo, em geral, em 27\% (OR = 0,73; IC95\%: 0,57-0.95) e sibilo atópico em 46\% (OR = 0,54; IC95\%: 0,30-0.98).

A Tabela 4 mostra o resultado da associação entre variáveis de consumo alimentar e asma em 
Associação entre grupos de alimentos/padrão alimentar de maior explicação da variabilidade da dieta e sibilo atópico e não atópico. Salvador, Bahia, Brasil 2005-2006.

\begin{tabular}{|c|c|c|c|c|c|c|c|c|c|c|c|c|}
\hline \multirow{2}{*}{$\begin{array}{l}\text { Grupos de } \\
\text { alimentos/Intervalo } \\
\text { do tercil }\end{array}$} & \multicolumn{4}{|c|}{ Geral * } & \multicolumn{4}{|c|}{ Atópico ** } & \multicolumn{4}{|c|}{ Não atópico *** } \\
\hline & OR \# & IC95\% & OR\#\# & IC95\% & OR \# & IC95\% & OR \#\# & IC95\% & OR \# & IC95\% & OR \#\# & IC95\% \\
\hline \multicolumn{13}{|l|}{ Pescados \#\#\# } \\
\hline T1 $(-0,10<-0,30)$ & 1,00 & - & 1,00 & - & 1,00 & - & 1,00 & - & 1,00 & - & 1,00 & - \\
\hline $\mathrm{T} 2(-0,30<0,18)$ & 0,72 & $0,55-0,93$ & 0,75 & $0,57-0,97$ & 0,86 & $0,53-1,41$ & 0,92 & $0,56-1,50$ & 0,62 & $0,47-0,84$ & 0,62 & $0,46-0,83$ \\
\hline T3 $(0,18-6,94)$ & 0,71 & $0,54-0,94$ & 0,73 & $0,56-0,94$ & 0,63 & $0,41-0,97$ & 0,75 & $0,48-1,15$ & 0,76 & $0,47-1,24$ & 0,69 & $0,42-1,11$ \\
\hline \multicolumn{13}{|l|}{ Vegetais/Tubérculos } \\
\hline $\mathrm{T} 1(-1,37<-0,46)$ & 1,00 & - & 1,00 & - & 1,00 & - & 1,00 & - & 1,00 & - & 1,00 & - \\
\hline $\mathrm{T} 2(-0,46<0,35)$ & 0,90 & $0,71-1,14$ & 0,93 & $0,71-1,22$ & 0,85 & $0,59-1,23$ & 0,91 & $0,62-1,35$ & 0,96 & $0,64-1,42$ & 0,94 & $0,62-1,43$ \\
\hline T3 $(0,35-5,1)$ & 0,94 & $0,68-1,30$ & 0,98 & $0,67-1,42$ & 0,64 & $0,38-1,06$ & 0,71 & $0,41-1,23$ & 1,27 & $0,83-1,96$ & 1,25 & $0,76-2,06$ \\
\hline \multicolumn{13}{|l|}{ Frutas } \\
\hline T1 $(-1,77<-0,52)$ & 1,00 & - & 1,00 & - & 1,00 & - & 1,00 & - & 1,00 & - & 1,00 & - \\
\hline T2 $(-0,52<0,33)$ & 1,00 & $0,70-1,44$ & 1,02 & $0,73-1,43$ & 0,93 & $0,68-1,27$ & 0,99 & $0,72-1,35$ & 1,11 & $0,67-1,84$ & 1,01 & $0,64-1,81$ \\
\hline T3 $(0,33-4,0)$ & 0,78 & $0,58-1,05$ & 0,8 & $0,56-1,15$ & 0,62 & $0,38-1,03$ & 0,75 & $0,40-1,40$ & 0,96 & $0,69-1,33$ & 0,85 & $0,56-1,29$ \\
\hline \multicolumn{13}{|l|}{ Leguminosas } \\
\hline T1 $(-0,64<-0,32)$ & 1,00 & - & 1,00 & - & 1,00 & - & 1,00 & - & 1,00 & - & 1,00 & - \\
\hline $\mathrm{T} 2(-0,32<0,33)$ & 0,80 & $0,59-1,11$ & 0,77 & $0,55-1,08$ & 0,81 & $0,55-1,19$ & 0,83 & $0,54-1,28$ & 0,72 & $0,43-1,21$ & 0,65 & $0,39-1,09$ \\
\hline T3 $(0,33-6,99)$ & 0,95 & $0,73-1,23$ & 0,93 & $0,70-1,22$ & 0,58 & $0,35-0,96$ & 0,62 & $0,35-1,10$ & 1,27 & $0,90-1,79$ & 1,13 & $0,80-1,60$ \\
\hline \multicolumn{13}{|l|}{ Cereais } \\
\hline $\mathrm{T} 1(-2,57<-0,46)$ & 1,00 & - & 1,00 & - & 1,00 & - & 1,00 & - & 1,00 & - & 1,00 & - \\
\hline T2 $(-0,46<0,37)$ & 1,01 & $0,79-1,42$ & 1,00 & $0,74-1,37$ & 1,36 & $0,99-1,87$ & 1,42 & $0,91-2,21$ & 0,99 & $0,68-1,43$ & 0,82 & $0,56-1,19$ \\
\hline T3 $(0,37-4,66)$ & 0,80 & $0,61-1,05$ & 0,80 & $0,54-1,19$ & 0,91 & $0,72-1,14$ & 0,87 & $0,50-1,51$ & 0,79 & $0,52-1,19$ & 0,69 & $0,39-1,23$ \\
\hline \multicolumn{13}{|l|}{ Padrão alimentar } \\
\hline $\mathrm{T} 1(-2,27<-0,48)$ & 1,00 & - & 1,00 & - & 1,00 & - & 1,00 & - & 1,00 & - & 1,00 & - \\
\hline $\mathrm{T} 2(-0,48<0,30)$ & 0,86 & $0,64-1,15$ & 0,83 & $0,62-1,09$ & 0,82 & $0,52-1,28$ & 0,81 & $0,51-1,29$ & 0,82 & $0,54-1,24$ & 0,76 & $0,48-1,19$ \\
\hline T3 $(0,30-5,19)$ & 0,75 & $0,58-0,97$ & 0,73 & $0,57-0,95$ & 0,49 & $0,30-0,81$ & 0,54 & $0,30-0,98$ & 0,98 & $0,71-1,37$ & 0,86 & $0,57-1,28$ \\
\hline
\end{tabular}

IC95\%: intervalo de 95\% de confiança; OR: odds ratio.

* Sibilo 12 meses;

** Sibilo 12 meses + IgE $\geq 0,70 K U / L$; Grupo de referência: crianças/adolescentes com apenas atopia (lgE $\geq 0,70 K U / L)$;

*** Sibilo 12 meses + IgE < 0,70KU/L; Grupo de referência: crianças/adolescentes sem sibilância;

\# Bruta;

\#\# Ajustado por sexo, idade da criança, indicador socioeconômico, asma dos pais, destino dos dejetos, água canalizada no domicílio, helmintos

(A. lumbricoides e T. trichiura), energia (Kcal), fumo no domicílio, idade da mãe, índice de massa corporal da criança;

\#\#\# Preparações à base de pescados.

geral, asma atópica e não atópica. O consumo de alimentos do grupo com preparação à base de pescados apresentou efeito protetor, reduzindo a ocorrência de asma, em geral, em $37 \%(\mathrm{OR}=0,63$; IC95\%: 0,47-0,83) e asma não atópica em 51\%, (OR = 0,49; IC95\%: 0.31-0,79). O maior tercil de consumo do padrão alimentar também reduziu a ocorrência de asma, em geral, em $36 \%(\mathrm{OR}=0,64$; IC95\%: 0,49-0,83) e asma atópica em 50\% (OR = 0,50; IC95\%: 0,28-0,89).

Foram realizadas associações entre os outros três padrões alimentares retidos na análise fatorial por componentes principais 18 , mas nenhum deles se mostrou associado a sibilo ou asma (dados não apresentados).

\section{Discussão}

O consumo de alimentos do grupo à base de pescados apresentou efeito protetor com redução da ocorrência de sibilo não atópico e asma não atópica. O padrão alimentar de maior variabilidade da dieta das crianças/adolescentes apresentou efeito protetor para a ocorrência de sibilo e asma atópica. 
Associação entre grupos de alimentos/padrão alimentar de maior variabilidade da dieta e asma atópica e não atópica. Salvador, Bahia, Brasil, $2005-2006$.

\begin{tabular}{|c|c|c|c|c|c|c|c|c|c|c|c|c|}
\hline \multirow{2}{*}{$\begin{array}{l}\text { Grupos de alimentos/ } \\
\text { Intervalo do tercil }\end{array}$} & \multicolumn{4}{|c|}{ Geral * } & \multicolumn{4}{|c|}{ Atópica ** } & \multicolumn{4}{|c|}{ Não atópica *** } \\
\hline & OR \# & IC95\% & OR \#\# & IC95\% & OR \# & IC95\% & OR \#\# & IC95\% & OR \# & IC95\% & OR \#\# & IC95\% \\
\hline \multicolumn{13}{|l|}{ Pescados \#\#\# } \\
\hline T1 $(-0,10<-0,30)$ & 1,00 & - & 1,00 & - & 1,00 & - & 1,00 & - & 1,00 & - & 1,00 & - \\
\hline T2 $(-0,30<0,18)$ & 0,73 & $0,53-1,00$ & 0,77 & $0,56-1,07$ & 0,83 & $0,48-1,44$ & 0,90 & $0,51-1,61$ & 0,66 & $0,46-0,95$ & 0,66 & $0,46-0,94$ \\
\hline T3 $(0,18-6,94)$ & 0,62 & $0,47-0,83$ & 0,63 & $0,47-0,83$ & 0,63 & $0,40-0,99$ & 0,77 & $0,46-1,28$ & 0,59 & $0,38-0,93$ & 0,49 & $0,31-0,79$ \\
\hline \multicolumn{13}{|l|}{ Vegetais/Tubérculos } \\
\hline T1 $(-1,37<-0,46)$ & 1,00 & - & 1,00 & - & 1,00 & - & 1,00 & - & 1,00 & - & 1,00 & - \\
\hline $\mathrm{T} 2(-0,46<0,35)$ & 0,90 & $0,66-1,24$ & 0,93 & $0,65-1,33$ & 0,88 & $0,57-1,37$ & 0,96 & $0,61-1,52$ & 0,92 & $0,57-1,48$ & 0,89 & $0,54-1,49$ \\
\hline T3 $(0,35-5,10)$ & 0,89 & $0,62-1,30$ & 0,92 & $0,60-1,41$ & 0,61 & $0,37-1,01$ & 0,68 & $0,39-1,19$ & 1,2 & $0,76-1,89$ & 1,15 & $0,68-1,94$ \\
\hline \multicolumn{13}{|l|}{ Frutas } \\
\hline $\mathrm{T} 1(-1,77<-0,52)$ & 1,00 & - & 1,00 & - & 1,00 & - & 1,00 & - & 1,00 & - & 1,00 & - \\
\hline $\mathrm{T} 2(-0,52<0,33)$ & 1,03 & $0,69-1,53$ & 1,05 & $0,72-1,53$ & 0,88 & $0,59-1,31$ & 0,93 & $0,62-1,38$ & 1,25 & $0,78-2,00$ & 1,23 & $0,77-1,96$ \\
\hline T3 $(0,33-4,00)$ & 0,84 & $0,61-1,16$ & 0,85 & $0,57-1,26$ & 0,61 & $0,36-1,02$ & 0,73 & $0,38-1,39$ & 1,12 & $0,77-1,64$ & 0,98 & $0,64-1,52$ \\
\hline \multicolumn{13}{|l|}{ Leguminosas } \\
\hline $\mathrm{T} 1(-0,64<-0,32)$ & 1,00 & - & 1,00 & - & 1,00 & - & 1,00 & - & 1,00 & - & 1,00 & - \\
\hline $\mathrm{T} 2(-0,32<0,33)$ & 0,78 & $0,59-1,03$ & 0,73 & $0,54-0,97$ & 0,80 & $0,55-1,16$ & 0,81 & $0,55-1,21$ & 0,67 & $0,39-1,14$ & 0,58 & $0,34-1,00$ \\
\hline T3 $(0,33-6,99)$ & 1,00 & $0,78-1,29$ & 0,95 & $0,71-1,25$ & 0,62 & $0,37-1,04$ & 0,67 & $0,38-1,19$ & 1,28 & $0,81-2,01$ & 1,08 & $0,70-1,68$ \\
\hline \multicolumn{13}{|l|}{ Cereais } \\
\hline $\mathrm{T} 1(-2,57<-0,46)$ & 1,00 & - & 1,00 & - & 1,00 & - & 1,00 & - & 1,00 & - & 1,00 & - \\
\hline $\mathrm{T} 2(-0,46<0,37)$ & 0,95 & $0,67-1,34$ & 0,89 & $0,62-1,29$ & 1,14 & $0,69-1,91$ & 1,18 & $0,74-1,89$ & 0,94 & $0,60-1,48$ & 0,81 & $0,50-1,30$ \\
\hline T3 $(0,37-4,66)$ & 0,77 & $0,60-1,00$ & 0,74 & $0,52-1,04$ & 0,62 & $0,41-0,94$ & 0,74 & $0,44-1,25$ & 0,88 & $0,57-1,35$ & 0,70 & $0,39-1,26$ \\
\hline \multicolumn{13}{|l|}{ Padrão alimentar } \\
\hline T1 $(-2,27<-0,48)$ & 1,00 & - & 1,00 & - & 1,00 & - & 1,00 & - & 1,00 & - & 1,00 & - \\
\hline $\mathrm{T} 2(-0,48<0,30)$ & 0,89 & $0,65-1,22$ & 0,84 & $0,62-1,15$ & 0,75 & $0,48-1,15$ & 0,74 & $0,47-1,15$ & 0,95 & $0,60-1,53$ & 0,89 & $0,54-1,45$ \\
\hline T3 $(0,30-5,19)$ & 0,69 & $0,54-0,90$ & 0,64 & $0,49-0,83$ & 0,46 & $0,29-0,75$ & 0,5 & $0,28-0,89$ & 0,90 & $0,60-1,35$ & 0,72 & $0,46-1,11$ \\
\hline
\end{tabular}

IC95\%: intervalo de $95 \%$ de confiança; OR: odds ratio.

* Sibilo nos últimos 12 meses + sintomas;

** Sibilo nos últimos 12 meses + sintomas + lgE $\geq 0,70 \mathrm{KU} / \mathrm{L}$; Grupo de referência: crianças/adolescentes com apenas atopia (lgE $\geq 0,70 \mathrm{KU} / \mathrm{L}$ );

*** Sibilo nos últimos 12 meses + sintomas + lgE <0,70KU/L; Grupo de referência: crianças/adolescentes não asmáticas;

\# Bruta;

\#\# Ajustado por sexo, idade da criança, indicador socioeconômico, asma dos pais, destino dos dejetos, água canalizada no domicílio, helmintos

(A. lumbricoides e T. trichiura), energia (Kcal), fumo no domicílio, idade da mãe, índice de massa corporal da criança;

\#\# Preparações à base de pescados.

Padrões alimentares similares aos encontrados em nossos achados têm sido identificados em outras populações e em outras faixas etárias por abarcarem alimentos comuns. Notável similaridade foi observada por Newby \& Tucker 31 revisando estudos que também derivaram padrões alimentares, em que foi notada a reprodutibilidade de um padrão com a presença de vegetais, frutas e legumes, caracterizando um padrão "prudente" ou "saudável". Tais alimentos estiveram presentes no padrão alimentar do presente trabalho, o qual contemplou também alimentos sugestivos da culinária local, a exemplo de azeite de dendê e leite de coco, correlacionados ao consumo de peixes, mariscos e crustáceos.
Essa mistura de alimentos presentes no grupo alimentar "pescados" pode reduzir a ocorrência de sintomas de asma em virtude de sua composição nutricional, envolvendo antioxidantes e ácidos graxos poli-insaturados, por refletir evidências relacionadas ao mecanismo fisiopatológico da asma 8,9 .

A hipótese lipídica abarca o mecanismo que prevê maior produção de mediadores inflamatórios (leucotrienos, tromboxanos, prostaglandinas) na medida em que se eleva a ingestão de ácidos graxos como aracdônico (C20:4n-6) e ácido linoléico (C18:2n-6) e se reduz o consumo de ácido graxo linolênico (C18:3n-3), ácido graxo eicosapentaenóico (EPA; C20:5n-3) e ácido do- 
cosaexaenóico (DHA; C22:6n-3), contribuindo para a ocorrência de sintomas de asma 9,32. Há vários achados na literatura que apoiam o papel protetor dos peixes para sintomas de asma e função respiratória $32,33,34$. Os frutos do mar e especialmente os peixes apresentam excelente relação ômega 6: ômega 3, sendo cerca de 1:1 a $2: 1$, para a maioria dos tipos de peixes, mariscos e crustáceos e há neles baixo teor de ácido aracdônico e gorduras saturadas 35 . Os peixes e frutos do mar são ótimas fontes de EPA e DHA 36 , ácidos graxos que exercem ainda potente efeito anti-inflamatório por meio do $G$ protein-coupled receptor 120 (GPR 120) 37 e do mediador lipídico anti-inflamatório resolvina E1 (RvE1) 38. Esses alimentos são ainda fontes de microminerais como zinco, cobre e selênio 35,36, os quais estão envolvidos em importantes mecanismos antioxidantes enzimáticos a exemplo da Glutationa Peroxidase e Superóxido Dismutase.

$\mathrm{O}$ azeite de dendê e o leite de coco não costumam ser componentes incorporados em grandes quantidades nas preparações típicas locais, comparados à presença dos peixes/mariscos/ crustáceos. Dessa forma, há uma possível desvantagem à hipótese lipídica para o azeite de dendê e leite de coco quanto à relação ômega 6: ômega 3, pode ser minimizada pela adição menor de ambos às preparações e pelos demais componentes nutricionais presentes, especialmente os antioxidantes do azeite de dendê (vitamina E, tocotrienois, beta carotenos, vitamina A, vitamina C) 39 e a presença de cobre e zinco no leite de coco 35 .

A hipótese antioxidante sinaliza que componentes antioxidantes da dieta a exemplo da vitamina A, carotenóides, vitamina C, vitamina $\mathrm{E}$, zinco, selênio, cobre e compostos bioativos podem conferir proteção ao sistema respiratório e reduzir sintomas de asma 9,10 na medida em que minimizam o processo oxidativo na resposta inflamatória 32,40 . Sabe-se que a ativação de células como neutrófilos, eosinófilos, monócitos e macrófagos na resposta inflamatória acontece sob condições oxidativas, resultando em um marcado aumento no consumo de oxigênio e consequente produção de espécies reativas de oxigênio, culminando no estresse oxidativo 32,41 . Nesse sentido, a proteção conferida em nossos achados pode ter minimizado o estresse oxidativo tanto na asma não atópica (grupo de pescados) quanto na asma atópica (padrão alimentar). O efeito antioxidante do padrão alimentar pode ter sido potencializado para asma atópica em função de concatenar maiores correlações para grupos alimentares fontes de antioxidantes. A combinação dos alimentos correlacionados no padrão alimentar deste estudo lembra componentes da dieta mediterrânea como peixes, frutas, vegetais, cereais em que o mais elevado nível de adesão a esta dieta tem sido protetor para ocorrência de asma 7,14 .

A patogenia da asma envolve ainda mecanismos complexos na modulação da resposta imune inata ou adquirida 12,40 . Na primeira, predomina um padrão neutrofílico na asma não atópica, com aumento nos níveis de IL-8, IL-6, ativação de receptores TLR4 e fator de transcrição fator nuclear kappa B (NF-kB); na segunda, predomina um padrão de eosinofilia na asma atópica, caracterizado pelo efeito alérgeno específico com diferenciação de linfócitos T na direção Th2 e envolvimento de citocinas IL-5, IL-13 e IL-4 e fator de transcrição GATA 12 .

Os resultados aqui encontrados com o consumo de pescados contribuem para explicação da asma não atópica no âmbito da resposta imune inata, variando de acordo com os componentes lipídicos e antioxidantes da dieta 12. Assim, ácidos graxos saturados iniciam resposta inflamatória inata pela ativação de receptores TLR4 42 e consequente ativação do NF-kB, desencadeando mecanismo inflamatório em cascata ${ }^{12}$. Na direção oposta, atuam os ácidos graxos poli-insaturados ômega 3, suprimindo atuação do NF-kB 42 e inativando receptores TLRs ${ }^{42}$. Com relação aos antioxidantes dietéticos, nota-se ativação do sistema imune inato com a vitamina $\mathrm{C}$ suprimindo a atividade do NF-kB 43; vitamina $\mathrm{E}$ reduzindo produção de TNF $\alpha$, IL-1 e IL-8 44; carotenóides sendo forte inibidor do NF-kB 45. A associação de vitaminas como E e C conferem inibição do NF-kB e espécies reativas de oxigênio, dentre outros aspectos 46 .

Assim, antioxidantes e lipídios dietéticos podem influenciar mecanismos imunomodulatórios e pró-inflamatórios complexos, com provável associação entre componentes nutricionais e parâmetros de asma 9,12.

A possibilidade de confundimento inerente ao alimento, perante a composição nutricional intrínseca ao próprio alimento e às combinações de alimentos dentro de um padrão alimentar, pode dificultar a identificação de aspectos da dieta mais diretamente implicados na doença. Sugerese a superação dessa limitação com a aplicação de métodos estatísticos e delineamentos que deem conta da questão intrínseca na direção de proteção e risco dentro de um mesmo alimento e dos alimentos entre si. Sugerem-se estudos futuros que considerem a imposição de aspectos teóricos prévios, em estatística multivariada confirmatória, a exemplo dos Modelos de Equações Estruturais.

O viés de memória na coleta de dados dietéticos é reconhecido há décadas na literatura. No 
entanto, Friedenreich et al. 47 e Willett 48 afirmam que os estudos conduzidos até hoje evidenciam que a dieta pode ser recordada com níveis aceitáveis de erro com intervalo de até aproximadamente dez anos, e que a maior preocupação são os estudos de casos e controles, cuja recordação pode ser diferencial e afetar a estimativa do risco relativo.

Importa destacar que nos resultados aqui encontrados há uma sincronia na direção dos achados, evidenciando faces da dimensão da dieta: seja mais ampla, com o resultado de associação do padrão alimentar; seja mais específica, com o resultado da associação dos grupos de alimentos estudados.

Abordar hábitos alimentares em uma população de um local específico é uma questão complexa, em virtude das profundas diferenças que vão desde as especificidades de cada região, influências culturais relacionadas à procedência da população e peculiaridades dos alimentos existentes. Não se pode deixar de considerar que os determinantes conjunturais da oferta, consumo e disponibilidade de alimentos, presentes na conjuntura de novos sistemas de vida na zona urbana, influenciam o consumo de alimentos.

\section{Conclusão}

Nossos achados sugerem que dieta caracterizada pelo consumo de peixes, mariscos e crustáceos, adicionada de itens da culinária local como azeite de dendê e leite de coco, pode conferir efeito protetor à ocorrência de sibilo e asma em crianças, especialmente para o fenótipo não atópico. Padrão alimentar caracterizado pelos alimentos anteriormente citados, além de frutas, vegetais, leguminosas e cereais reduz a ocorrência de asma atópica e estudos futuros devem demonstrar a sua efetividade na redução da asma.

\section{Resumen}

Este estudio transversal se llevó a cabo para estudiar la influencia de los hábitos alimentarios en la aparición del asma y sibilancias atópicas y no atópicas en una muestra de 1.168 niños y adolescentes en Salvador, Bahía, Brasil. Las sibilancias y síntomas de asma en los últimos 12 meses se han obtenido a partir del cuestionario ISAAC. Se identificó la presencia de IgE específica de alérgeno aéreo. Se utilizó un cuestionario de frecuencia de alimentos para definir hábitos alimentarios. Se aplicó la regresión logística y el modelo logístico multinomial. El consumo de pescado redujo las sibilancias en un 27\% (IC95\%: 0,56-0,94) y el asma en un 37\% (IC95\%:
0,47-0,83), las sibilancias atópicas en un 51\% (IC95\%: 0,31-0,79) y no atópicas en un 38\% (IC95\%: 0,46-0,83). El tercil más alto de los hábitos alimentarios reduce si bilancias en un 27\% (IC95\%: 0,57-0,95), sibilancias atópicas en un 46\% (IC95\%: 0,30-0,98), el asma en un 36\% (IC95\%: 0,49-0,83) y un 50\% en el asma atópico (IC95\%. 0,28-0,89). El consumo de pescado puede promover un efecto protector, dentro el patrón de dieta, contra sibilancias atópicas y no atópicas y asma.

Conducta Alimentaria; Asma; Ruidos Respiratorios 


\section{Colaboradores}

S. D'Innocenzo foi responsável pela redação do manuscrito, participou na coleta de dados dietéticos, realizou análise estatística e contribuiu na revisão final do manuscrito. S. M. A. Matos, M. S. Prado e A. M. O. Assis participaram da coleta dos dados e revisão do documento. C. A. S. T. Santos contribuiu com as análises estatísticas e revisão do manuscrito. A. A. Cruz e D. M. L. Marchioni contribuíram com a revisão do manuscrito. L. C. Rodrigues e M. L. Barreto participou no planejamento e financiamento do estudo e revisão do manuscrito. Todos os autores revisaram e aprovaram a versão final do manuscrito.

\section{Referências}

1. Asher MI. Recent perspectives on global epidemiology of asthma in childhood. Allergol Immunopathol 2010; 38:83-7.

2. Cooper PJ, Rodrigues LC, Barreto ML. Influence of poverty and infection on Latin America. Curr Opin Allergy Clin Immunol 2012; 12:171-8.

3. Solé D, Wandalsen GF, Camelo-Nunes IC, Naspitz CK. Prevalence of symptoms of asthma, rhinitis, and atopic eczema among Brazilian children and adolescents identified by the International Study of Asthma and Allergies in Childhood (ISAAC) Phase 3. J Pediatr 2006; 82:341-6.

4. Simões SM, Cunha SS, Barreto ML, Cruz AA. Distribution of severity of asthma in childhood. J Pediatr 2010; 86:417-23.

5. Cunha SS, Barreto ML, Fiaccone RL, Cooper PJ, Alcantara-Neves NM, Simões SM, et al. Asthma cases in childhood attributed to atopy in tropical area in Brazil. Rev Panam Salud Pública 2010; 28:405-11.

6. Brandão HV, Cruz CMS, Junior ISS, Ponte EV, Guimarães A, Cruz AA. Hospitalizações por asma: impacto de um programa de controle de asma e rinite alérgica em Feira de Santana (BA). J Bras Pneumol 2009; 35:723-9.

7. Cooper PJ, Rodrigues LC, Cruz AA, Barreto ML. Asthma in Latin America: a public health challenge and research opportunity. Allergy 2009; 64: 5-17.

8. Litonjua AA. Dietary factors and the development of asthma. Immunol Allergy Clin North Am 2008; 28:603-29.

9. Devereux G, Seaton A. Diet as risk factor for atopy and asthma. J Allergy Clin Immunol 2005; 115:1109-17.

\section{Agradecimentos}

Às famílias das crianças/adolescentes que participaram deste estudo. Ao Instituto de Saúde Coletiva/Universidade Federal da Bahia, no âmbito do SCAALA (Social Changes, Asthma and Allergy in Latin America). Ao The Wellcome Trust e CNPq.

10. Allen S, Britton JR, Leonardi-Bee JA. Association between antioxidant vitamins and asthma outcome measures: systematic review and meta-analysis. Thorax 2009; 64:610-9.

11. Garcia-Marcos L, Castro-Rodriguez JA, Weinmaryr G, Panagiotakos DB, Priftis KN, Nagel G. Influence of Mediterranean diet on asthma in children: a systematic review and meta-analysis. Pediatr Allergy Immunol 2013; 24:330-8.

12. Wood LG, Gibson PG. Dietary factors lead to innate immune activation in asthma. Pharmacol Ther 2009; 123:37-53.

13. Chatzi L, Kogevinas M. Prenatal and childhood Mediterranean diet and the development of asthma and allergies in children. Public Health Nutr 2009; 12:1629-34.

14. Nagel G, Weinmayr G, Kleiner A, Garcia-Marcos L, Strachan DP. Effect of diet on asthma and allergic sensitization in the International Study on Allergies and Asthma in Childhood (ISAAC) Phase Two. Thorax 2010; 65:516-22.

15. Hu FB. Dietary pattern analysis: a new direction in nutritional epidemiology. Curr Opin Lipidol 2002; 13:3-9.

16. Willett WC. Nutritional epidemiology. New York: Oxford University Press; 1998.

17. Jacobs DRJ, Steffen LM. Nutrients, foods, and dietary patterns as exposures in research: a framework for food synergy. Am J Clin Nutr 2003; 78 Suppl 3:508S-13.

18. Barros R, Moreira A, Fonseca J, Oliveira JF, Delgado L, Castel-Branco MG, et al. Adherence to the Mediterranean diet and fresh fruit intake are associated with improved asthma control. Allergy 2008; 63:917-23. 
19. Barreto ML, Cunha SS, Alcântara-Neves N, Carvalho LP, Cruz AA, Stein RT, et al. Risk factors and immunological pathways for asthma and other allergic diseases in children: background and methodology of a longitudinal study in a large urban center in Northeastern Brazil (Salvador-SCAALA study). BMC Pulm Med 2006; 23:6-15.

20. Asher MI, Keil U, Anderson HR, Beasley R, Crane J, Martinez F, et al. International study of asthma and allergies in childhood (ISAAC): rationale and methods. Eur Respir J 1995; 8:483-91.

21. D’Innocenzo S, Marchioni DML, Prado MS, Matos SMA, Pereira SR, Barros AP, et al. Condições socioeconômicas e padrões alimentares de crianças de 4 a 11 anos: estudo SCAALA - Salvador/ Bahia. Rev Bras Saúde Matern Infant 2011; 11:41-9.

22. Matos SMA, Prado MS, Santos CAST, D'Innocenzo S, Assis AMO, Rodrigues LC, et al. Validation of food frequency questionnaire for children and adolescents aged 4 to 11 years living in Salvador, Bahia. Nutr Hosp 2012; 27:1114-9.

23. Andrade RG, Pereira RA, Sichieri R. Consumo alimentar de adolescentes com e sem sobrepeso do Município do Rio de Janeiro. Cad Saúde Pública 2003; 19:1485-95.

24. WHO Multicentre Growth Reference Study Group. WHO Child Growth Standards based on length/ height, weight and age. Acta Paediatr 2006; 450: 76-85.

25. de Onis M, Onyango A, Borghi E, Siyam A, Nishida C, Siekmann J. Development of a WHO growth reference for school-aged children and adolescents. Bull World Health Organ 2007; 85:661-8.

26. Neva FA, Brown HW. Basic clinical parasitology. $6^{\text {th }}$ Ed. Norwalk: Appleton \& Lange; 1994.

27. Katz N, Chaves A, Pellegrino J. A simple device for quantitative determination of Schistosoma mansoni eggs in faeces examined by the thick-smear technique. Rev Inst Med Trop São Paulo 1972; 14: 394-400.

28. Alvarez ML, Muzzo S, Ivanovic D. Escala para medición del nivel socioeconómico, en el área de la salud. Rev Med Chile 1985; 113:243-9.

29. Issler RMS, Giugliani ERJ. Identificação de grupos mais vulneráveis à desnutrição infantil pela medição do nível de pobreza. J Pediatr (Rio J.) 1997; 73:101-5.

30. Hosmer D, Lemeshow S. Applied logistic regression. 2nd Ed. New York: John Wiley and Sons; 2000.

31. Newby PK, Tucker KL. Empirically derived eating patterns using factor or cluster analisys: a review. Nutr Rev 2004; 62:177-203.

32. Burns JS, Dockery DW, Neas LM, Schwartz J, Coull BA, Raizenne M, et al. Low dietary nutrient intakes and respiratory health in adolescents. Chest 2007; 132:238-45.

33. Tabak C, Wijga AH, de Meer G, Janssen NA, Brunekreef B, Smit HA. Diet and asthma in Dutch school children (ISAAC-2). Thorax 2006; 61: 1048-53.
34. Oddy WH, de Klerk NH, Kendall GE, Mihrshahi S, Peat JK. Ratio of omega-6 omega-3 fatty acids and childhood asthma. J Asthma 2004; 41:319-26.

35. Núcleo de Estudos e Pesquisas em Alimentação, Universidade Estadual de Campinas. Tabela brasileira de composição de alimentos - TACO. 2a Ed. Campinas: Núcleo de Estudos e Pesquisas em Alimentação, Universidade Estadual de Campinas; 2006.

36. Mahan LK, Escott-Stump S. Alimentos, nutrição \& dietoterapia. 11ạ Ed. São Paulo: Roca; 2005.

37. Oh Y, Talukdar S, Bae EJ, Imamura T, Morinaga $\mathrm{H}$, Fan W, et al. GPR120 is an omega-3 fatty acid receptor mediating potent anti-inflamatory and insulin-sensitizing effects. Cell 2010; 142:687-98.

38. Aoki $\mathrm{H}$, Hisada $\mathrm{T}$, Ishizuka $\mathrm{T}$, Utsugi $\mathrm{M}$, Ono $\mathrm{A}$, Koga Y, et al. Protctive effect of Resolvin E1 on the development of asthmatic airway inflammation. Biochem Biophys Res Commun 2010; 400:128-33.

39. Coordenação-Geral da Política de Alimentação e Nutrição, Secretaria de Políticas de Saúde, Ministério da Saúde. Alimentos regionais brasileiros. Brasília: Ministério da Saúde; 2002. (Série F. Comunicação e Educação em Saúde, 21).

40. Holgate S. Pathogenesis of asthma. Clin Exp Allergy 2008; 38:872-97.

41. Nadeem A, Masood A, Siddiqui N. Oxidant-antioxidant imbalance in asthma: scientific evidence, epidemiological data and possible therapeutic options. Ther Adv Respir Dis 2008; 2:215-35.

42. Lee JY, Sohn KH, Rhee SH, Hwang D. Saturated fatty acids, but not unsaturated fatty acids, induce the expression of cyclooxygenase- 2 mediated through Toll-like receptor 4. J Biol Chem 2001; 276:16683-9.

43. Hartel C, Strunk T, Bucsky P, Schultz C. Effects of vitamin $\mathrm{C}$ on intracytoplasmic cytokine production in human whole blood monocytes and lymphocytes. Cytokine 2004; 27:101-6.

44. Van Tits LJ, Demacker PN, De Graaf J, Hak-Lemmers HL, Stalenhoef AF. Alpha-tocopherol supplementation decreases production of superoxide and cytokines by leukocytes ex vivo in both normolipidemic and hypertriglyceridemic individuals. Am J Clin Nutr 2000; 71:458-64.

45. Li BH, Zhang QX, Dong DJ, Lin XM. Effect of lycopene on immunity in rats with acute lung injury. Beijing Da Xue Xue Bao 2007; 39:77-82.

46. Tan PH, Sagoo P, Chan C, Yates JB, Campbell J, Beutelspacher SC, et al. Inhibition of NF-kB and oxidative pathways in human dendritic cells by antioxidative vitamins generates regulatory $\mathrm{T}$ cells. J Immunol 2005; 174:7633-44.

47. Friedenreich CM, Slimani N, Riboli E. Measurement of past diet: review of previous and proposed methods. Epidemiol Rev 1992; 14:177-96.

48. Willett W. Nutritional epidemiology. 3rd Ed. New York: Oxford University Press; 2013.

Recebido em 18/Set/2013

Versão final reapresentada em 05/Fev/2014

Aprovado em 17/Fev/2014 